\title{
Towards a Resource-Centric Data Network for Astronomy
}

\author{
Alberto Accomazzi ${ }^{* \dagger}$ \\ Harvard-Smithsonian Center for Astrophysics \\ E-mail: aaccomazzi@cfa.harvard.edu

\section{Michael J. Kurtz} \\ Harvard-Smithsonian Center for Astrophysics \\ E-mail: mkurtz@cfa.harvard.edu
}

\section{Stephen S. Murray}

Harvard-Smithsonian Center for Astrophysics

E-mail: ssmecfa.harvard.edu

\begin{abstract}
Over the past decade, astronomers have been using an increasingly larger number of web-based applications and archives to conduct their research. However, despite the early success in creating links across projects and data centers, the promise of a single integrated digital library environment supporting e-science in astronomy has proven elusive. While some of the issues hampering progress in this area are of technical nature, others are rooted in existing policies which should be re-analyzed if further rapid progress is to be made in this area. This paper describes a proposal that the NASA Astrophysics Data System project has put forth in order to improve its role as one of the primary discovery portals for astronomers, focusing on those aspects which could benefit from an increased level of involvement from the community, namely the effort to expose astronomy resources as linked data, and the harvesting of observational metadata.
\end{abstract}

Accelerating the Rate of Astronomical Discovery, sps5

August 11-14, 2009

Rio de Janeiro, Brazil

\footnotetext{
*Speaker.

$\dagger$ This work was supported by the Astrophysics Data System project which is funded by NASA grant NNX09AB39G
} 


\section{Introduction}

Astronomy is an observational science. Our theories of the behavior of the universe and the objects in it are inspired by observations of that behavior, and are supported (or rejected) by observations, as well. Within the past twenty years systems of dense, interlinked data have become common and expected, fundamentally changing the way people think and services operate. It is now expected, for example, that from a listing of a movie at our local theater one can find a synopsis, reviews, a cast list, a list of all the director's films, recommendations, and the option to purchase tickets, as well.

Astronomy was one of the first disciplines to benefit from the early developments of webbased technologies enabling cross-linking of resources across archives (Accomazzi et al, 1994). Sixteen years ago, thanks to a collaboration between the NASA Astrophysics Data System (ADS) and the Centre de Données Astronomiques de Strasbourg (CDS), it became possible to go from a list of articles to the abstract of an article to a list of astronomical objects described in that article to a set of measurements for each of those objects. Thirteen years ago, again thanks to a collaboration between the ADS and several major data centers, including NED, HEASARC, MAST, ESO, and Chandra, it became possible to go from an article abstract to the actual observational data used to write the article, and then back to all publications describing each observation.

These connections have enabled astronomers to use the search capabilities of any of the main archives to locate a dataset or publication of interest, and then follow the appropriate links to find related information provided by another archive. For instance, using ADS, one could search for papers on X-rays emission in Abell clusters and filter results to obtain just papers which have links to data products. While this is a very useful way to narrow bibliographic searches, selecting which of these papers has links to images or spectra or catalogs still requires a person to click through all the data links provided in the list of returned papers. Automating this activity is currently not practical since connections between one archive and another are purely defined as links between URLs, and lack semantic and contextual information between the resources they represent. In this paper we argue that a tighter integration of observational and bibliographic metadata will enable the creation of new connections between resources in astronomy and applications that will allow users to search, browse, and reason over them.

The existence of these connections between and within papers and data products represents more that just a convenient feature to the end-user. The research process in science today involves the generation, retrieval, manipulation, analysis, and publication of digital artifacts which are typically stored in different archives on the web. In order to enable scientists to access these scholarly products, and to document and recreate the analysis that was carried out on them, a formal description of these resources and processes is necessary (Pepe et al., 2009). Creating these descriptions and establishing relationships and links among them provides a way to satisfy a number of different goals, among them: aggregation of related resources (linking together all artifacts used in a study, including data products, notes, draft papers, software tools); attribution (properly acknowledging prior work, tools being used, or datasets being analyzed); preservation (maintaining provenance information and reconstructing the workflow used in the research process); and discovery (following connections between resources may uncover previously unknown relationships). 


\section{A Model for Interlinking Resources}

A model for describing and interlinking resources is provided by the Linked Data Effort ${ }^{1}$, which is based on the Resource Description Framework (RDF) model (W3C, 2009): a resource has properties which have values. For example $<$ PaperA $><$ isWrittenBy $><$ Author $X>$ or $<$ PaperA $>$ $<$ isCitedBy $><$ PaperB $>$. Chaining these relationships together and performing functions on the results yields what we call Second Order Operators (Kurtz, 1992). These operations can have very powerful properties. For example, chaining $<$ wordPhrase $><$ is ContainedIn $\rangle<$ Papers $X>$ with $<$ Papers $X><$ areCitedBy $><$ Papers $Y>$ yields a list of papers which cite papers which contain the phrase. Sorting this list by frequency of appearance yields review articles on the original word phrase, which could be any topic such as "dark energy," or "extrasolar planets." The ADS has been internally making use of the relationships between bibliographic metadata records since its inception to provide some of its more advanced retrieval capabilities.

An example of what is possible to achieve exploiting these relationships is provided by the myADS notification service (Kurtz et al. 2003). Originally introduced in 2003, this service now delivers customized email or RSS updates to over 7,000 users providing them with updates about the recent technical literature in astronomy and physics. One of the features included in the update are the most popular and most cited papers on two topics of interest to the subscriber. Computation of both lists makes use of citation and usage relationships data which interlink bibliographic records in ADS. Another example of a service that makes use of these operators is ADS's topic search', which can be used to search the literature for a topic in different ways. This includes returning a list of the most popular, useful and instructive papers on a particular subject. In this context, "popular," "useful," and "instructive" have been defined in terms of second order operations on bibliographic, citation, and usage data.

Observational metadata may be similarly modeled: an observation has an observing proposal, a position in the sky, a time of observation, an instrument, a telescope, an observer, and a P.I., among other properties. These properties will likewise have attributes: the observing proposal will contain words, and may contain a list of observations; the P.I. may have written papers describing such observations; the position on the sky may correspond with a known object; the instrument will have a type (e.g. imager, spectrograph) and settings (filter, wavelength range, resolution); a combination of time, position and telescope will yield air mass and moon phase, and so on.

A brief example will illustrate how this knowledge can be used to facilitate a typical researcher's workflow. Given a list of the positions of extragalactic globular clusters one could search for all observations which are IR spectra. Downloading and analyzing the CaII triplet in these spectra would allow one to research the metallicity distribution in the nearby universe. The links from the observing proposals for these spectra to the journal articles, as well as the proposals themselves, would allow the user to determine the original intent of the observations, and whether the proposed work had, indeed, already been done. This new system of observation interlinking would clearly be tightly integrated with the existing infrastructure. For example, the list of observations of IR spectra of extragalactic globular clusters will link to a list of observing proposals, which will link to a set of journal articles which will have been cited by other journal articles, and so on.

\footnotetext{
${ }^{1} \mathrm{http}: / /$ linkeddata.org

${ }^{2} \mathrm{http}$ ://adsabs.harvard.edu/ads_abstracts.html
} 
More in general, adopting Linked Data practices to identify, describe, and connect resources which are already available in our archives provides us with a simple way to expose, share, and link pieces of data, information, and knowledge about them. One advantage of this design is that it enables the creation of a global graph, i.e. a web of resources which reside in different locations but which are connected by semantic relationships. The very existence of the graph make it possible for agents and applications to traverse it in order to describe parts of it, create new connections within it, or compute new results from it.

\section{Infrastructure and Applications}

While the bibliographic metadata in Astronomy is currently available from a single access point (the ADS), the observational metadata is stored by a number of archives and projects in different formats. Bibliographic searches and Data searches are now being carried out in separate domains, even though links between the ADS and the archives allow one to go back and forth between papers and data products. However, to date there is no application which efficiently combines a bibliographic search with a "data" search based on observational parameters (such as instrument, wavelength, etc) across archives.

An obvious way to enable a more advanced discovery system is to expose the observational and instrument metadata maintained by the different archives. Much of this content already exists, having been created and curated by groups such as the CDS, ESO, and the NASA archives and data centers, but the metadata describing this content is currently unavailable for harvesting in machinereadable format. Exposing this observational and technical metadata at the appropriate granularity level would enable a number of useful applications to be build. For example, the ADS project could enhance its holdings by harvesting metadata on observational resources which are necessary to interlink observations and publications more tightly. Other organizations would be able to take advantage of the availability of this metadata as well. For instance, an institute maintaining a list of publications by its staff could more easily find out what type of datasets have been used by them in their research efforts. A researcher starting a new project could identify the core papers on the subject area and find all datasets studied by them.

A simple prototype interface recently developed by the ADS provides a good illustration of what types of applications become easily possible once observational and bibliographic resources become better interlinked. The interface in question builds upon the topic search mentioned in the earlier section and adds to it a view of the search results by related astronomical objects. Figure 1 illustrates an example of the results that are generated from such an interface. Suppose a researcher wants to know what are the extrasolar planets that are most often referenced in review papers. Using the topic search interface, the user can request the list of most instructive papers on the topic of "extrasolar planets." As results from the query are retrieved, the system queries the SIMBAD database to find the list of objects mentioned in each of the papers. The cumulative list of these object names is then ranked by how frequently each of them appears in the original set of papers, and is displayed on the left bar. This list represents a set of so-called "facets" which can be used to further drill into search results, or provide additional views of the information that is buried within them and otherwise difficult to see. Using this additional information, a researcher can quickly identify which objects are most relevant to the topic of interest. Applications can also associate 


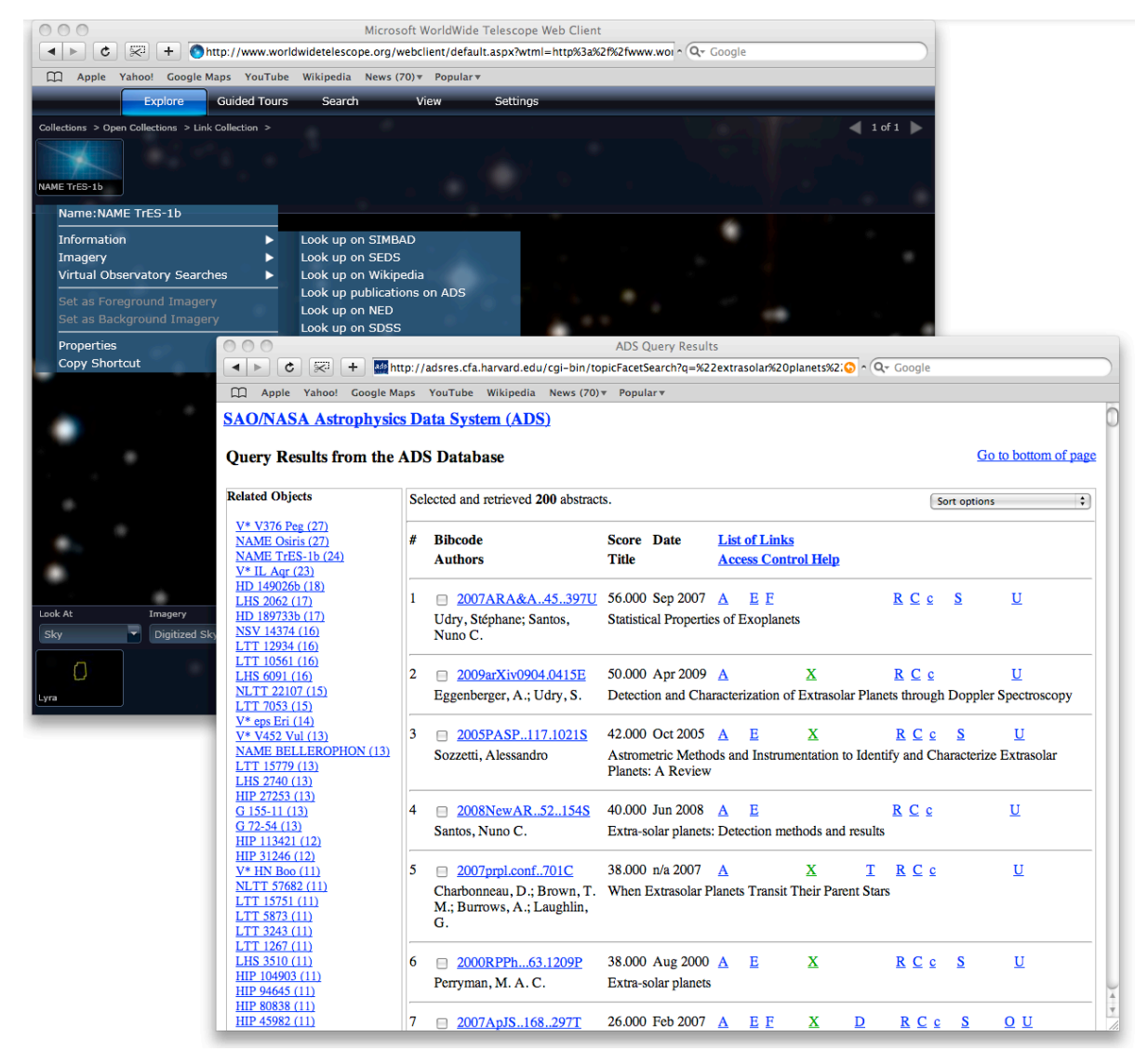

Figure 1: A prototype application implementing object-based facets as a view into search results. The original search requested review articles about "extrasolar planets." From the list of most relevant objects, the third entry from the top was selected (TrES-1b), and the object was displayed via a link to the WWT web client.

one or more actions to each set of faceted views. In the example shown in Figure 1, we have provided the option to display each object via the WorldWideTelescope web client application, but one could have chosen to use any number of SAMP-enabled applications to display or map one or more of the objects in question.

It's easy to imagine a variety of similar scenarios in which bibliographic or observational properties are used to create similar facets. For example, when manipulating a list of papers, facets could be built on the sets of keywords that are shared amongst them, or the type of data products associated with them. While not all facets may make sense in all cases, there are certainly several scenarios in which they provide insightful views into the data. We hope that in the coming years we will be able to investigate the usefulness and impact of faceted views of the literature when combined with additional observational metadata.

\section{Discussion}

In this coming era of data intensive science, it is increasingly important for researchers to be 
able to seamlessly move between scientific results, the data used to publish them, and the processes used to produce them. In addition, scientific research requires that we are able to establish the provenance of data sources and processes operating on this data, so that research may be repeated, variations on the research be carried out, and new research on existing datasets be enabled.

While the Virtual Observatory has provided us with the standards and protocols needed to model and exchange astronomical datasets, the problem of information discovery in our datacentric world still looms large. The key to creating such a system is having access to the metadata describing the datasets at the appropriate granularity level so that the proper resources can be identified, described, and connections between them can be made. The technology which can be used to expose and link these metadata has now matured and is the foundation of the semantic web framework and in particular in the linked data model. These technologies promise to become widely-adopted standards enabling the creation and growth of a "web of data" in which machinereadable content parallels the human-readable content available today.

We believe that the astronomical community would benefit from embracing this philosophy and adopting these technologies. In particular, three sets of metadata resources currently stored in our archives should be made available machine-readable format for harvesting and indexing: observing proposals, their attributes and links to observations; observations and their attributes; instruments, their attributes and capabilities. From a technical standpoint this metadata should be exposed as RDF and should make use of community-developed lightweight ontologies. This will allow the use of linked-data principles to properly connect these resources. However, even before these technical aspects are worked out, we believe that the availability of the metadata to organizations such as the ADS, the CDS, NED, and other IVOA members will foster the creation of knowledge bases and applications demonstrating the value of such an interlinked data system. Therefore we urge the community to support this effort by recommending that observatories and archives make available and permit the harvesting and indexing of metadata describing publicly accessible datasets, including observing proposals, observing logs, and FITS headers.

\section{References}

[1] Accomazzi, A., Grant, C., Eichhorn, G., Kurtz, M., \& Murray, S., The World Wide Web and ADS services, Bulletin of the American Astronomical Society, 26, 1370 (1994)

[2] Kurtz, M.J., Second Order Knowledge: Information Retrieval in the Terabyte Era, in: Astronomy from Large Databases II. European Southern Observatory Proceedings, A. Heck and F. Murtagh, eds., 85 (1992)

[3] Kurtz, M. J., Eichhorn, G., Accomazzi, A., Grant, C. S., Henneken, E. A., Thompson, D. M., Bohlen, E. H., \& Murray, S. S., The myADS Update Service, Bulletin of the American Astronomical Society, $35,1241(2003)$

[4] Pepe, A., Mayernik, M., Borgman, C. L., \& Van de Sompel, H., From Artifacts to Aggregations: Modeling Scientific Life Cycles on the Semantic Web [arXiv:0906.2549] (2009).

[5] W3C, Resource Description Format (RDF), http://www.w3.org/RDF/ (2009) 